above study, SPECT was abnormal in all patients and remained abnormal at followup.

Cerebral vein thrombosis as a complication of SLE was reported in 3 girls admitted with persistent headache at the Hospital for Sick Children, Toronto (Uziel Y et al. LPediatr 1995;126:722-727; see Progress in Pediatric Neurology III, PNB Publ, 1997;173-4).

Autoimmune-mediated, childhood onset obsessive-compulsive disorder and tics are the subject of a review (Hamilton CS, Swedo SE Clinical Neuroscience Research Jan 2001;1:61-68) from the National Institute of Mental Health, Bethesda, MD. In this subgroup of PANDAS there is a temporal association between Group A Beta Hemolytic Streptococcal infection and onset or exacerbation of symptoms. If a causal link can be confirmed, antibiotic treatment may be justified as an adjunct therapy of OCD and tics.

SPECT findings in early-onset OCD. Regional cerebral blood flow (rCBF) SPECT was measured in 13 early-onset $(<10$ years) and 13 late-onset $(>12$ years) adult OCD subjects and in 22 healthy controls, in a study at the University of Sao Paulo Medical School, Brazil. Early-onset OCD subjects showed decreased rCBF in the right thalamus, left anterior cingulate, and both inferior prefrontal cortices relative to late-onset cases $(\mathrm{p}<.0005)$, and severity of OCD symptoms corresponded with left orbitofrontal rCBF. The mechanisms involved in OCD may differ according to age at onset of symptoms. (Busatto GF et al. LAm Acad Child Adolesc Psychiatry March 2001;40:347-354).

\title{
ISOLATED ANGIITIS OF THE CNS
}

The clinical features, pathology, and outcome of isolated (primary, idiopathic) angiitis of the CNS (LACNS) in two new and 8 previously reported cases are analysed at the Hospital for Sick Children, Toronto, Canada. Cases were grouped according to the size of arteries affected - 1) small or 2) large and medium:

Group 1. IACNS affected small vessels in 5 cases, including the 2 new cases. Onset of symptoms was gradual, with persistent headaches in 3 , multifocal neurologic deficits (2), cognitive impairment (1), mood disorder (1), and focal seizures (3). Brain MRI was abnormal in all 5, showing a single tumor-like mass in 2. Angiography was abnormal in only 1, showing stenosis of a quaternary branch of the middle cerebral. Diagnosis was confirmed by CNS biopsy, showing a nongranulomatous (lymphocytic) vasculitis. Immunosuppression treatment with prednisone was successful in 4 who are alive without recurrence; 1 died at 18 months after presentation, despite the addition of cyclophosphamide in thgerapy.

Group 2. Large artery IACNS in 5 cases presented with ischemic stroke in 2, transient ischemic attacks (1), and subarachnoid hemorrhage (2). Four died within 10 days, and the fifth child had cerebral hematomas secondary to recurrent ruptured aneurysms. The wall of one resected aneurysm showed inflammatory infiltration, and this child died 7 years after presentation. All 5 at autopsy showed granulomatous infiltration and necrosis of large and medium vessels. Elevated ESR, inflammatory CSF, and abnormal angiograms are more frequent in group 2 patients. Clinical evaluation excluded other causes, including drug exposures, systemic infection, thromboembolism, and rheumatic disease. Patients with poor outcome had involvement of large and medium-sized arteries, presentation with acute stroke, granulomatous angiitis on brain biopsy, and 
delayed treatment with prednisone. (Lanthier S, Lortie A, Michaud J et al. Isolated angiitis of the CNS in children. Neurology April (1 of 2) 2001;56:837-842). (Reprints: Dr Gabrielle deVeber, Division of Neurology, The Hospital for Sick Children, 555 University Avenue, Toronto, Ontario, Canada, M5G 1X8).

COMMENT. Isolated, primary angiitis of the CNS in children may affect either small or large arteries. The clinical and radiological manifestations correlate with the size of vessel involved, and the outcome differs between groups. A poor outcome is more likely in cases presenting with acute stroke and disease of large arteries, granulomatous angiitis on biopsy, and delay in instituting immunosuppressive therapy. CNS involvement by systemic infection or rheumatic disease, including lupus erythematosus, should be considered in angiitis affecting small cerebral vessels.

\section{INFECTIOUS DISORDERS}

\section{LA CROSSE ENCEPHALITIS}

The clinical manifestations and course of La Crosse encephalitis were studied in 127 school-aged children (mean age, 7.8 years; range, 6 months - 15 years) hospitalized from 1987 through 1996 at the Robert C Byrd Health Sciences Center, West Virginia University, Charleston, WVa. Serologic testing was positive for IgM and IgG antibodies to La Crosse virus. Viral cultures of CSF were negative. Headache, fever, and vomiting were the most common presenting symptoms, each occurring in $70 \%$ or more of patients. Seizures occurred in $46 \%$, and disorientation in $42 \%$. Aseptic meningitis was present in $13 \%$, signs of increased intracranial pressure in $13 \%$ ( 3 with cerebral herniation), and hyponatremia developed in $21 \%$, consistent with the syndrome of inappropriate antidiuretic hormone secretion. EEGs were abnormal in 59 of 90 patients with recordings; 29 had focal features with temporal lobe involvement, suggestive of herpes simplex encephalitis. Fever resolved in $70 \%$ of patients after 3 days, and the mean stay in hospital was 6 days. Risk factors for clinical deterioration and need for intervention, affecting $11 \%$ of patients, were hyponatremia, fever, vomiting, seizures, and a score of 12 or lower on the Glasgow Coma Scale on admission. All survived, but $12 \%$ had neurologic sequelae, including cognitive and behavior deficits (ADHD in 15 patients), at 10 to 18 month follow-up. (McJunkin JE, de los Reyes EC, Irazuzta JE et al. La Crosse encephalitis in children. N Fngl I Med March 15, 2001;344:801-807). (Reprints: Dr McJunkin, Department of Pediatrics, West Virginia University, PO Box 9214, Morgantown, WV 26506).

COMMENT. La Crosse virus, a member of the California encephalitis serogroup, was first isolated from the brain of an affected child who died in La Crosse County, Wisconsin, and was reported in 1965. A mosquito-borne disease, and the most prevalent childhood arboviral infection in $\mathrm{N} \mathrm{America,} \mathrm{is} \mathrm{often} \mathrm{misdiagnosed}$ as enteroviral meningitis or herpes simplex encephalitis. The disease is highly endemic in the Midwest and in West Virginia, with a reported incidence of 20-30 cases per 100,000 children. Hyponatremia is a common complication and a risk factor for seizures and clinical deterioration. Cognitive impairment and ADHD are frequent neurobehavioral sequelae, similar to the reports following the World War 1 influenza epidemic and encephalitis of 1918. (Ebaugh F. Am I Dis Child 1923;25:89-97; see Millichap JG. Attention Deficit Hyperactivity \& Learning Disorders. PNB Publ, 2001). 